\title{
Stability Analysis of Nonlinear Singular Systems via Polytopic Models of the Characteristic Equation *
}

\author{
Marcelino Sánchez* Juan Carlos Arceo* Victor Estrada-Manzo* \\ Miguel Bernal* \\ * Dept. of Electrical and Electronics Engineering, Sonora Institute of \\ Technology, 5 de Febrero 818 Sur, C.P. 85000, Cd. Obregon, Sonora, Mexico, \\ miguel.bernal@itson.edu.mx
}

\begin{abstract}
This report explores asymptotic stability of nonlinear singular systems, i.e., differential algebraic equations with a descriptor state-space representation, by means of a polytopic rewriting of the "generalised characteristic polynomial" (determinant of the corresponding nonlinear pencil). It is shown that, via linearisation arguments, the Edge Theorem can be adapted for analysis purposes by taking into account that singularity of systems translates into degree dropping of some vertex polynomials.
\end{abstract}

Keywords: Nonlinear Singular Systems, Linear Matrix Inequalities, Characteristic Polynomial.

\section{INTRODUCTION}

State-space forms of singular nonlinear systems are descriptors (Dai, 1989):

$$
E(x) \dot{x}(t)=A(x) x(t),
$$

where $x(t) \in \mathbb{R}^{n}$ is the state, $A(x), E(x) \in \mathbb{R}^{n \times n}$ are smooth matrix nonlinear functions, and, importantly, $E(x)$ has not full rank. Simulation of these systems requires proper initialisation to hold the algebraic restrictions along the time (Brenan et al., 1995); moreover, sudden loss of rank in $E(x)$ may lead to instantaneous novel algebraic restrictions which might be consistent/inconsistent, persistent/evitable. Simulation of DAEs is largely based on the Pantelides algorithm in Pantelides (1988) which, under certain conditions, transforms it into an ordinary differential equation (ODE). Since we are interested in stability of equilibrium points, in the sequel, it will be assumed that $x=$ 0 is an equilibrium point of (1). Lyapunov-based analysis of such systems is still on course, based on generalised Lyapunov functions, descriptor redundancy forms, and/or the Finsler's Lemma (Ishihara and Terra, 2002); only particular cases are available in the nonlinear case: when explicit algebraic restrictions are provided (Ebenbauer and Allgöwer, 2007), the rank of $E(x)$ is fixed (quasi-linear) (Riaza and Zufiria, 2001), or $E(x)$ is constant (Yang et al., 2013).

This paper adopts a different perspective: it considers matrix $s E(x)-A(x)$ as a generalised pencil and $\operatorname{det}(s E(x)-A(x))$ as the generalised characteristic polynomial associated to (1); based on them, stability is established by mimicking eigenvalue tests from linear systems theory. Since (1) is not a linearparameter- nor a time-varying system, guaranteeing that the roots of $\operatorname{det}(s E(x)-A(x))$ are always in the left half of the complex plane $\mathbb{C}^{-}$is enough to ensure asymptotic stability of the origin by linearisation arguments. To check the eigenvalue condition for a given a compact subset of the state space $\Omega \subset \mathbb{R}^{n}$, our proposal rewrites $\operatorname{det}(s E(x)-A(x))$ as a polytope of vertex polynomials; then, a variety of tests derived from

\footnotetext{
$\star$ The authors would like to thank the support provided by the CONACYT scholarships 423601 and 415714, the postdoctoral fellowship for CVU 366627, the ITSON PROFAPI Project CA-18 2017-0088, and the PFCE 2016-17.
}

the Edge Theorem (Bartlett et al., 1988) come at hand to provide sufficient conditions for the polytope to be stable; these tests are expressed in the form of linear matrix inequalities (LMIs) (Boyd et al., 1994). Importantly, the singular nature of the systems is mirrored by degree dropping of some vertex polynomials, which obliges to recur to further refinements of the Edge Theorem (Białas and Góra, 2012).

\section{POLYTOPES OF POLYNOMIALS}

A bounded expression can always be written as a convex sum of its bounds; indeed, given $z \in\left[z^{0}, z^{1}\right]$, it can be algebraically checked that $z=w_{0}(z) z^{0}+w_{1}(z) z^{1}$ with $w_{0}(z)=$ $\left(z^{1}-z\right) /\left(z^{1}-z^{0}\right)$ and $w_{1}(z)=1-w_{0}(z)$; therefore, assuming all non-constant terms $z_{j}(x) \in\left[z_{j}^{0}, z_{j}^{1}\right], j \in\{1,2, \ldots, r\}$ in the coefficients of $\operatorname{det}(s E(x)-A(x))$ are bounded in $\Omega$, where $z_{j}^{0}=\min _{x \in \Omega} z(x)$ and $z_{j}^{1}=\max _{x \in \Omega} z(x)$, they can be written as

$$
z_{j}(x)=\underbrace{\left(\frac{z_{j}^{1}-z_{j}(x)}{z_{j}^{1}-z_{j}^{0}}\right)}_{w_{0}^{j}(x)} z_{j}^{0}+\underbrace{\left(\frac{z_{j}(x)-z_{j}^{0}}{z_{j}^{1}-z_{j}^{0}}\right)}_{w_{1}^{j}(x)} z_{j}^{1},
$$

where $w_{0}^{j}(x)+w_{1}^{j}(x)=1,0 \leq w_{i}^{j}(x) \leq 1, i \in\{0,1\}$ for any $x \in \Omega$ (convex sum property).

Once this is made, $\operatorname{det}(s E(x)-A(x))$ can be rewritten as a polytope of polynomials in $s$; each of these vertexes has constant coefficients which correspond to a particular combination of minima and maxima of the $r$ non-constant terms in $\operatorname{det}(s E(x)-A(x))$. Let $\mathbf{i}=\left(i_{1}, i_{2}, \ldots, i_{r}\right), \forall j: i_{j} \in\{0,1\}$, $\mathbf{w}_{\mathbf{i}}(z)=w_{i_{1}}^{1}(z) w_{i_{2}}^{2}(z) \cdots w_{i_{r}}^{r}(z)$; then:

$$
\operatorname{det}(s E(x)-A(x))=\sum_{\mathbf{i}} \mathbf{w}_{\mathbf{i}}(z) p_{\mathbf{i}}(s),
$$

where $\sum_{\mathbf{i}} \mathbf{w}_{\mathbf{i}}(z)=\sum_{i_{1}=0}^{1} \sum_{i_{2}=0}^{1} \cdots \sum_{i_{r}=0}^{1} w_{i_{1}}^{1}(z) w_{i_{2}}^{2}(z) \cdots w_{i_{r}}^{r}(z)=$ $1,0 \leq \mathbf{w}_{\mathbf{i}}(z) \leq 1, p_{\mathbf{i}}=\left.\operatorname{det}(s E(x)-A(x))\right|_{\mathbf{w}_{\mathbf{i}}=1}$. Since $\operatorname{rank}(E(x))<n$ certain vertex polynomials $p_{\mathbf{i}}(s)$ will present degree dropping. 
Example 1. Consider the nonlinear singular system

$$
\left[\begin{array}{cc}
\left(x_{1}-x_{2}\right)^{2} & 0 \\
0 & 1
\end{array}\right]\left[\begin{array}{l}
\dot{x}_{1} \\
\dot{x}_{2}
\end{array}\right]=\left[\begin{array}{cc}
1 & -1 \\
-1-x_{1}^{2} & -2
\end{array}\right]\left[\begin{array}{l}
x_{1} \\
x_{2}
\end{array}\right],
$$

whose generalised characteristic polynomial is

$$
\left(x_{1}-x_{2}\right)^{2} s^{2}+\left(2\left(x_{1}-x_{2}\right)^{2}-1\right) s-x_{1}^{2}-3 .
$$

As expected, sudden loss of rank in the left-hand side of (4) and degree dropping of (5) occur when $x_{1}-x_{2}=0$. An exact polytopic rewriting of (5) for the compact set $\Omega$ where $z_{1}(x)=\left(x_{1}-x_{2}\right)^{2} \in[0, \alpha]$ and $z_{2}(x)=x_{1}^{2} \in[0, \beta]$ with $\alpha, \beta>0$, can be done by writing (3) with $p_{00}(s)=-s-3$, $p_{01}(s)=-s-\beta-3, p_{10}(s)=\alpha s^{2}+(2 \alpha-1) s-3$, and $p_{11}(s)=\alpha s^{2}+(2 \alpha-1) s-\beta-3$ as vertex polynomials, and $w_{0}^{1}(z)=\left(\alpha-\left(x_{1}-x_{2}\right)^{2}\right) / \alpha, w_{0}^{2}(z)=\left(\beta-x_{1}^{2}\right) / \beta, w_{1}^{1}=1-w_{0}^{1}$, and $w_{1}^{2}=1-w_{0}^{2}$ as convex interpolating functions. Again, degree dropping occurs in vertex polynomials $p_{00}(s)$ and $p_{01}(s)$ as a result of the system singularities.

\section{POLYNOMIAL-BASED STABILITY}

Let $H(\cdot) \in \mathbb{R}^{n \times n}$ denote the Hurwitz matrix of a polynomial $p(s)=a_{n} s^{n}+a_{n-1} s^{n-1}+\cdots+a_{1} s+a_{0}$. Since the seminal paper of Białas (1985), sufficient and necessary conditions for the stability of convex combinations of two $n$-th degree stable polynomials have been established by constructing an "edge-like" test which examines whether the real eigenvalues of the composite matrix $H_{12}=-H\left(p_{1}(s)\right) H^{-1}\left(p_{2}(s)\right)$ are strictly negative, where $p_{1}(s)=s^{n}+a_{n-1} s^{n-1}+\ldots+a_{1} s+a_{0}$ and $p_{2}(s)=s^{n}+b_{n-1} s^{n-1}+\ldots+b_{1} s+b_{0}$ are the stable polynomials in the convex combination $\lambda p_{1}(s)+(1-\lambda) p_{2}(s), \lambda \in[0,1]$. This criterium has been put into an LMI form for polytopes of stable polynomials (Sánchez and Bernal, 2017). When degree dropping of some vertex polynomials occur, refinements exist that are based on further considerations on the geometry of the complementary regions (Białas and Góra, 2012).

Theorem 1. Assume the origin $x=0$ of the nonlinear singular system (1) is an equilibrium point. Assume also that the system has a generalised characteristic polynomial with exact convex representation (3) in the compact set $\Omega$. Then, the origin $x=0$ is asymptotically stable in the sense of singular systems if there exists matrices $M_{\mathrm{ij}}$ of adequate dimension, such that LMIs

$$
M_{\mathrm{ij}}+M_{\mathrm{ij}}^{T}>0, H\left(p_{\mathbf{i}}\right) M_{\mathrm{ij}} H^{T}\left(p_{\mathbf{j}}\right)+H\left(p_{\mathbf{j}}\right) M_{\mathbf{i j}}^{T} H^{T}\left(p_{\mathbf{i}}\right) \geq 0,
$$

are feasible for all $\mathbf{i}, \mathbf{j} \in\{0,1\}^{r} \operatorname{such}$ that $\operatorname{deg}\left(p_{\mathbf{j}}\right) \geq \operatorname{deg}\left(p_{\mathbf{i}}\right)$, $\mathbf{i} \neq \mathbf{j}$, provided each vertex polynomial $p_{\mathbf{i}}$ is stable.

Proof. If conditions (6) hold, then $M_{\mathrm{ij}}+M_{\mathrm{ij}}^{T}>0$ and $H^{-1}\left(p_{\mathbf{j}}\right) H\left(p_{\mathbf{i}}\right) M_{\mathbf{i j}}+M_{\mathbf{i j}}^{T} H^{T}\left(p_{\mathbf{i}}\right) H^{-T}\left(p_{\mathbf{j}}\right) \geq 0$ hold, which implies that the real eigenvalues of each $H^{-1}\left(p_{\mathbf{j}}\right) H\left(p_{\mathbf{i}}\right)$ are all nonnegative (Ebihara and Onishi, 2009). This, in turn, implies that the convex sum (3) is a polytope of stable polynomials with possibly degree dropping in some of its vertexes (Białas and Góra, 2012). Recall that the convex sum (3) is algebraically equivalent to $p(s, x)=\operatorname{det}(s E(x)-A(x))$ for all $x \in \Omega$, which therefore implies that there is a $P \in \mathbb{R}^{n \times n}$ such that $E^{T}(0) P=P^{T} E(0) \geq 0$ and $P A(0)+A^{T}(0) P \leq 0$. Thus, a linearisation argument allows using $V(x)=x^{T} E^{T}(0) P x$ as a Lyapunov function for all the trajectories in a sufficiently small vicinity within a subset $\mathcal{X} \subset \mathbb{R}^{n}$ with $\operatorname{dim}(\mathcal{X}) \leq \operatorname{rank}(E(0))$, to which the dynamics of the system are restricted, i.e., asymptotic stability of the origin $x=0$ in the sense of singular systems is granted.
Example 1 (continued): Consider again the nonlinear singular system (4) in example 1, whose characteristic polynomial is given in (5). Modelling as shown thereby with $\alpha=100$ and $\beta=25$ leads to four polynomials $p_{00}(s)=-s-3, p_{01}(s)=-s-$ $28, p_{10}(s)=100 s^{2}+199 s-3$, and $p_{11}(s)=100 s^{2}+199 s-28$, where the last two are unstable. This implies the test is unable to establish stability in this region, which is consistent with the fact that it includes unstable trajectories. Notwithstanding, since $E(x)$ loses rank in $x_{1}-x_{2}=0$ leading to a persistent algebraic restriction which includes the origin, the generalised characteristic polynomial can be reduced to $p(s, x)=-s-3+$ $x_{1}^{2}$ which, taking $z_{2}(x)$ can be modelled as the convex sum of $p_{0}(s)=-s-3$ and $p_{1}(s)=-s-28$ whose associated Hurwitz matrices yield feasible LMI conditions in Theorem 1 with

$$
H\left(p_{0}\right)=\left[\begin{array}{cc}
-1 & 0 \\
0 & -3
\end{array}\right], H\left(p_{1}\right)=\left[\begin{array}{cc}
-1 & 0 \\
0 & -28
\end{array}\right], M_{12}=\left[\begin{array}{cc}
0.5053 & 0 \\
0 & 0.0061
\end{array}\right] \text {. }
$$

This result confirms that stable trajectories are indeed those in the algebraic restriction $x_{1}-x_{2}=0$ where $E(x)$ loses rank.

\section{REFERENCES}

Bartlett, A.C., Hollot, C.V., and Lin, H. (1988). Root locations of an entire polytope of polynomials: It suffices to check the edges. Mathematics of Control, Signals and Systems, 1(1), $61-71$.

Białas, S. (1985). A necessary and sufficient condition for the stability of convex combinations of stable polynomials or matrices. Bulletin of the Polish Academy of Sciences, 33(910), 473-480.

Białas, S. and Góra, M. (2012). A few results concerning the hurwitz stability of polytopes of complex polynomials. Linear Algebra and its Applications, 436(5), 1177-1188.

Boyd, S., Ghaoui, L.E., Feron, E., and Belakrishnan, V. (1994). Linear Matrix Inequalities in System and Control Theory, volume 15. SIAM: Studies In Applied Mathematics, Philadelphia, USA.

Brenan, K.E., Campbell, S.L., and Petzold, L.R. (1995). Numerical solution of initial-value problems in differentialalgebraic equations. SIAM.

Dai, L. (1989). Singular Control Systems. Springer-Verlag.

Ebenbauer, C. and Allgöwer, F. (2007). Stability analysis of constrained control systems: An alternative approach. Systems $\mathcal{E}$ Control Letters, 56(2), 93-98.

Ebihara, Y. and Onishi, Y.and Hagiwara, T. (2009). Robust performance analysis of uncertain LTI systems: Dual LMI approach and verifications for exactness. IEEE Transactions on Automatic Control, 54(5), 938-951.

Ishihara, J.Y. and Terra, M. (2002). On the Lyapunov theorem for singular systems. IEEE Transactions on Automatic Control, 47(11), 1926-1931.

Pantelides, C.C. (1988). The consistent initialization of differential-algebraic systems. SIAM Journal on Scientific and Statistical Computing, 9(2), 213-231.

Riaza, R. and Zufiria, P.J. (2001). Stability of singular equilibria in quasilinear implicit differential equations. Journal of Differential Equations, 171(1), 24-53.

Sánchez, M. and Bernal, M. (2017). A convex approach for reducing conservativeness of kharitonovs-based robustness analysis. IFAC-PapersOnLine, 50(1), 832-837.

Yang, C., Sun, J., and Zhang, Q.and Ma, X. (2013). Lyapunov stability and strong passivity analysis for nonlinear descriptor systems. IEEE Transactions on Circuits and Systems I: Regular Papers, 60(4), 1003-1012. 